
toujours pertinentes?

\title{
Les associations de médecins : désuètes ou toujours pertinentes?
}

Gerald B. Brock, MD, FRCSC

Président de l'AUC

Cite as: Can Urol Assoc J 2017; Epub ahead of print. http://dx.doi.org/10.5489/cuaj.5040

\section{Published online November 1, 2017}

\section{$* * *$}

Au milieu des années 50, 75 \% de tous les médecins exerçant aux États-Unis étaient membres de l’American Medical Association. De nos jours, selon les données, ce chiffre se situe sous les $15 \%$ et continue de chuter. Est-ce le montant de la cotisation, le manque de pertinence? Ou y a-t-il tout simplement trop d’associations, de groupes de spécialités et de réunions? C’est dans l'air du temps : l’idée de se joindre à de larges groupes ou organisations a perdu de son lustre pour bien des gens.

À l’Association des urologues du Canada, nous reconnaissons que nous devons mériter l'appui de nos membres au jour le jour en fournissant des services et des programmes qui répondent à vos besoins sans coûter cher ni exiger beaucoup de votre temps. Nous savons que nous sommes en concurrence avec de nombreuses autres sociétés et associations de médecins et de spécialités à votre établissement, dans votre région et dans votre province.

C’est dans cet esprit que nous avons travaillé fort au cours des derniers mois à l'évaluation des activités actuelles du très efficace et très productif Bureau de l’éducation. Pour ce faire, nous avons procédé à une évaluation des besoins de nos membres, discuté avec nos commanditaires de l’industrie et formulé un plan stratégique officiel. L’objectif clair à long terme est de cerner les thèmes importants qui ont le plus d'impact sur les 
Les associations de médecins : désuètes ou toujours pertinentes?

urologues et de fournir des activités de développement professionnel continu d'avantgarde. La direction empruntée par le Collège royal vers la détermination des compétences de nos résidents en formation débouchera probablement sur des exigences similaires pour les urologues exerçant leur profession. Dans cette perspective, nous nous affairons à créer, de manière proactive, des initiatives éducatives conçues pour nos membres afin d’être en mesure d’anticiper toute exigence éducative future.

Notre réunion d’hiver, tenue chaque année en janvier à Montréal, arrive à grands pas. Lors de cette réunion, les dirigeants passent en revue nos activités et fixent les objectifs de l'association pour les prochains mois. Nous espérons également à cette occasion discuter de vos commentaires à vous, les membres, concernant les services et les produits que vous souhaitez voir développer. N’hésitez pas à faire part par courriel à Tiffany Pizioli, notre directrice exécutive (Tiffany.Pizioli@cua.org), moi-même (President@cua.org),ou tout autre dirigeant de l’association (http://www.cua.org/en/about-cua) de ce que vous aimeriez que nous fassions pour vous aider dans votre travail ou quel service vous aimeriez voir ajouté ou modifié.

Nous mettons actuellement la touche finale au programme du congrès annuel d’Halifax, où nous vous présenterons un excellent programme interactif offert par un superbe groupe de conférenciers de partout dans le monde, notamment :

- Benjamin Canales, M. D., MPH (Gainesville, Floride), professeur agréé d’urologie, Département d’urologie, École de médecine de l’Université de la Floride

- Jamie Campbell, M. Ed., B. Ed., B. A. (Hon.) (Toronto, Ont.), directeur, Services de développement organisationnel et de bénévolat, Hôpital général de North York 
Les associations de médecins : désuètes ou toujours pertinentes?

- Robert Flanigan, M. D., FACS (Maywood, Ill.), professeur et chaire Albert J. Speh Jr. et Claire R. Speh, Département d’urologie, École de médecine Stritch de l’Université Loyola à Chicago; président de l’AUA

- Freddie Hamdy, M. D. (Oxford, Angleterre), Université d’Oxford, professeur Nuffield de chirurgie; chef, Département Nuffield des sciences chirurgicales; professeur d'urologie; consultant honoraire et chirurgien urologique; Fellow de Balliol College

- Venkata Jayanthi, M. D. (Columbus, Ohio), chef, urologie pédiatrique, Nationwide Children’s Hospital; professeur clinique de chirurgie, Département d’urologie, Faculté de médecine de l’Université d’État de l’Ohio

- Lorraine LeGrand-Westfall, M. D., FRCSC, ACPM (Ottawa, Ont.), directrice des affaires régionales; agente principale, Protection de la vie privée - Association canadienne de protection médicale

- Anne Pelletier Cameron, M. D., FRCSC (Ann Arbor, Mich.), professeure agréée d’urologie, Université du Michigan, directrice de CURE (Clinical Urology Research Endeavor)

- Hossein Sadeghi-Nejad, M. D., FACS (Newark, New Jersey), professeur de chirurgie/urologie, École de médecine Rutgers du New Jersey; chef, Division d’urologie, New Jersey Veterans Affairs Hospitals; directeur, Center for Male Reproductive Medicine (cabinet privé), Centre médical universitaire Hackensack

- Joel Sheinfeld, M. D. (New York, NY), chef adjoint, Service d’urologie, chaire Florence et Theodore Baumritter/Enid Ancell d’uro-oncologie, Centre de traitement du cancer Memorial Sloan-Kettering 
Les associations de médecins : désuètes ou toujours pertinentes?

- Run Wang, M. D., FACS (Houston, Texas), professeur de chirurgie (urologie) et Cecil M. Crigler, chaire MD d’urologie, Faculté de médecine de l’Université du Texas; professeur d'urologie, directeur, Division de médecine sexuelle, Département d’urologie, Centre de cancérologie MD Anderson de l’Université du Texas - président de la SMSNA

- Brian Goldman, M. D. (Toronto, Ont.), animateur et médecin

Au plaisir de vous voir à Halifax! 